\title{
Acoustic measurement of under-expanded jet and its numerical prediction
}

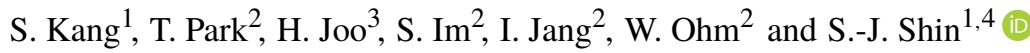 \\ ${ }^{1}$ Seoul National Univerity, Department of Aerospace Engineering, Seoul, Republic of Korea, ${ }^{2}$ Yonsei Univerity, Department of \\ Mechanical Engineering, Seoul, Republic of Korea, ${ }^{3}$ Korea Shipbuilding and Offshore Engineering, Innovative Machinery \\ Systems Research Department, Seongnam, Republic of Korea and ${ }^{4}$ Seoul National Univerity, Institute of Advanced Aerospace \\ Technology, Seoul, Republic of Korea \\ Email: ssjoon@snu.ac.kr
}

Received: 23 August 2021; Revised: 25 November 2021; Accepted: 6 January 2022

Keywords: Aeroacoustics; Jet noise; Acoustic measurement; Vertical ejection; Numerical simulation; Deflector

\begin{abstract}
Intense acoustic loads from jet noise cause noise pollution and induce failures, such as the malfunctioning of electronic devices and fatigue failure of internal/external structures. Consequently, the prediction of jet noise characteristics is crucial in the development of high-speed vehicles. This study presents acoustic experiments and predictions for an under-expanded, unheated jet using a small-scale prototype. Outdoor measurements are carried out using a vertical ejection setup. Acoustic characteristics are measured using both linear and circular microphone arrays. Additionally, numerical prediction of the same jet noise is performed using a detached eddy simulation and the permeable Ffowcs-Williams and Hawkings acoustic analogy. The vertical experimental setup exhibits the typical acoustic characteristics of a supersonic jet in terms of directivity and broadband shock-associated noise. Moreover, the numerical prediction exhibits satisfactory accuracy for the jet downstream, where the large-scale turbulence structures of the directivity predominate. However, discrepancy increases in the domain of lower directivity. The presented experiment and prediction will be extended to future studies regarding the noise of various deflector duct configurations impinging on supersonic jets.
\end{abstract}

\section{Nomenclature}

A area

$A^{*} \quad$ area of the nozzle throat

c speed of sound

D diameter

$f \quad$ frequency

$L \quad$ loading noise vector

M Mach number

$\mathbf{M}_{c o} \quad$ Oertel's convective Mach number

$\hat{n} \quad$ unit outward normal vector

$p \quad$ pressure

$p^{\prime} \quad$ acoustic pressure in time domain

$\hat{p}^{\prime} \quad$ Fourier-transformed acoustic pressure

$P \quad$ compressive stress tensor

$Q \quad$ observer position vector

Re Reynolds number

$s \quad$ distance between observer and source

$\hat{s} \quad$ unit vector in the radiation direction

St Strouhal number

( $)$ The Author(s), 2022. Published by Cambridge University Press on behalf of Royal Aeronautical Society. This is an Open Access article, distributed under the terms of the Creative Commons Attribution licence (http://creativecommons.org/licenses/by/4.0/), which permits unrestricted re-use, distribution, and reproduction in any medium, provided the original work is properly cited. 
$T \quad$ static temperature

$u \quad$ flow velocity vector

$U \quad$ flow speed

axial distance from the nozzle exit

\section{Greek symbol}

$\delta$

$\Delta$

$\gamma$

$\omega$

$\rho$

$\theta$

\section{Subscripts}

\section{$a$}

c

$e$

$j$

ret

0

\author{
Kronecker delta \\ grid spacing \\ specific heat ratio \\ angular frequency \\ density \\ angle between the jet plume and microphone
}
physical quantities at the atmosphere
physical quantities related to the cut-off frequency
physical quantities at the nozzle exit condition
physical quantities related to fully expanded jet condition
physical quantities evaluated at the retarded time
physical quantities at the stagnation condition

\subsection{Introduction}

The jet and rocket engines of a high-speed vehicle provide a significant amount of thrust, which is accompanied by excessive acoustic loads emitted from the nozzle. Jet noise exhibits unique characteristics, such as high amplitude, large uncertainty, and broadband spectra. In particular, the jet noise becomes more severe in the supersonic regime with additional complicated elements, such as screech tones and broadband shock-associated noise [1]. Crackle is another shock-related component that induces an irritating noise by nonlinear propagation [2,3]. In addition to noise pollution, intense acoustic loads from the jet noise will induce failures [4], such as the malfunction of vehicle electronic devices and fatigue failure of the internal/external structures. Therefore, the prediction of jet noise characteristics has emerged as a priority task during the development of high-speed vehicles. Furthermore, the design process should include an accurate prediction of the structural responses induced by acoustic loads.

Since the studies by Lighthill $[5,6]$, characteristics of supersonic jet noise have been extensively investigated over the past few decades $[1,7,8]$. Based on the knowledge and understanding of supersonic jet noise, empirical/semi-empirical procedures for noise have been developed and methodologies have been established. NASA SP-8072 [9], which became a foundation for high-speed rocket noise, proposed an empirical prediction method (the distributed sound source allocation method) based on the compilation of the lift-off acoustic results of the sound source. Through several modifications, the NASA SP-8072 method has been consistently adopted, owing to its simplicity [10-14]. Haynes and Kenny [10] predicted the lift-off noise of ARES-I and suggested modifications of the second distributed source method (DSM-II). The modifications of DSM-II, which were originally included in NASA SP8072, were completed based on the characteristic length formulations of the jet core revised by Varnier [11], concepts of the core termination, and the new noise directivity index. Kandula [12] conducted near-field acoustic prediction for clustered rocket engines based on NASA SP-8072, including ground reflection. Barbarino et al. [13] proposed an explicit expression for the acoustic pressure of each sound source while indicating an uncorrelated source for the definition of the sound pressure level (SPL) in NASA SP-8072. Semi-empirical methods have been continuously developed and are still in use, owing to their simplicity and computational costs; however, they are insufficient for a few cases and cannot concretely demonstrate the complex mechanism of the noise with high fidelity. In contrast, full-scale acoustic experiments may be regarded as one of the most direct methods for noise evaluation. However, 
(a)

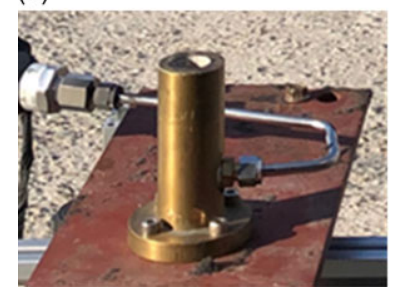

(b)

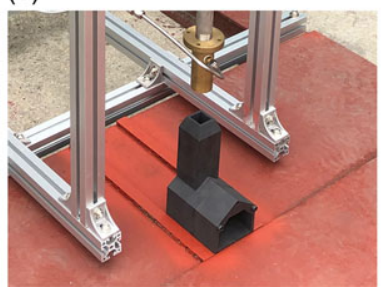

(c)

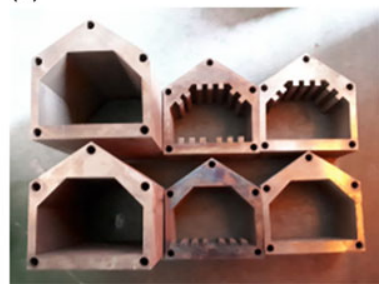

Figure 1. Small-scale prototypes. (a) Nozzle. (b) Deflector. (c) Deflector ducts of various designs.

moderate alternatives, such as numerical prediction and small-scale experiments, are preferred, owing to cost constraints.

Moreover, as the capability of high-performance computing increased, noise prediction via numerical simulations became more feasible. Most numerical simulations for acoustic analysis rely on computational fluid dynamics (CFD), more specifically, the large eddy simulation (LES), including both zonal/non-zonal hybrid models of Reynolds-averaged Navier-Stokes (RANS) equations and the LES [14-20]. To validate the results, several numerical simulations were attempted against small-scale acoustic experiments, full-scale tests, and flight results. In many cases, studies on small-scale testing were evaluated with regard to their similarity to full-scale models [19, 21, 22]. The acoustic spectra regarding the Strouhal number became less dependent on the Reynolds number [23], particularly at high Reynolds numbers, which enabled small-scale modeling. Fukuda et al. [14] employed an implicit LES to predict the acoustic mechanism of the static firing experiments of an NAL-735 rocket motor, and their predictions exhibited better correlation with the measurements at the resolved frequencies compared with the methods given in NASA SP-8072. Ishii et al. [24] experimentally investigated the noise of a 1/42-scale booster and the launch pad of an Epsilon launch vehicle. Measurements were conducted for various deflector designs and flight altitudes for the near/far-field using phased array microphones. Tsutsumi et al. [18] carried out 1/42-scale acoustic measurements for Epsilon launch pads under various conditions. They also conducted a numerical investigation for the largest acoustic loads using zonal hybrid RANS/LES CFD. The same researchers [19] performed both numerical simulations and acoustic measurements for launch pads with flame ducts, and they compared the acoustic levels with the full-scale flight results of Epsilon-1. An implicit LES and zonal hybrid RANS/LES were adopted for the preliminary and CFD simulations, respectively. Kiris et al. [20] introduced several implementations of the launch, ascent, and vehicle aerodynamics framework, which combined CFD capabilities with conjugate heat transfer and computational aeroacoustics modules on the NASA space launch system. Malbéqui et al. [25] conducted an acoustic measurement on a 1/40-scale launch pad based on the preliminary design of Ariane 6. Jiang et al. [26] summarised the research efforts that were accumulated prior to 2019 regarding acoustic prediction and experiments on the rocket launch.

The present authors have previously conducted investigations regarding the noisy environment surrounding high-speed vehicles via small-scale testing prototypes, such as those depicted in Fig. 1 [27-29]. This study presents both experimental measurements and numerical predictions for the noise of an unheated, under-expanded supersonic free jet in a small-scale test. Outdoor measurements of jet noise are conducted using linear and circular microphone arrays for the near- and far-field noise, respectively. Vertical ejection setup is employed for the flow generation, which is expected to avoid ground reflection by intense Mach wave radiation. Qualitative and qualitative measurements are obtained with sufficient reproducibility. For the numerical aspect, a three-dimensional detached eddy simulation (DES), which is a non-zonal RANS/LES model, is executed to predict the jet noise, and the results are compared with those of the experiments. This study also investigates if the proposed experimental and numerical setups are capable of manifesting the main characteristics of supersonic jet noise. In Section 2, the experimental setups of vertical ejection and acoustic measurement systems are described. The obtained result is validated in terms of reproducibility, and compared against the similarity spectra. Section 3 explains 
Table 1. Operating condition of the small-scale nozzle

\begin{tabular}{lcc}
\hline Operating Condition & & Value \\
\hline Nozzle Exit Diameter & $D_{e}$ & $20[\mathrm{~mm}]$ \\
Nozzle Designed Mach Number & $\mathrm{M}_{d}$ & 1.81 \\
Fully Expanded Jet Mach Number & $\mathrm{M}_{j}$ & 1.88 \\
Fully Expanded Jet Temperature & $T_{j}$ & $176[\mathrm{~K}]$ \\
Nozzle Exit Reynolds Number & $\mathrm{Re}_{e}$ & $1.8 \times 10^{6}$ \\
Nozzle Area Ratio & $A_{e} / A^{*}$ & 1.445 \\
Nozzle Pressure Ratio & $p_{0} / p_{a}$ & 6.5 \\
\hline
\end{tabular}

(a)

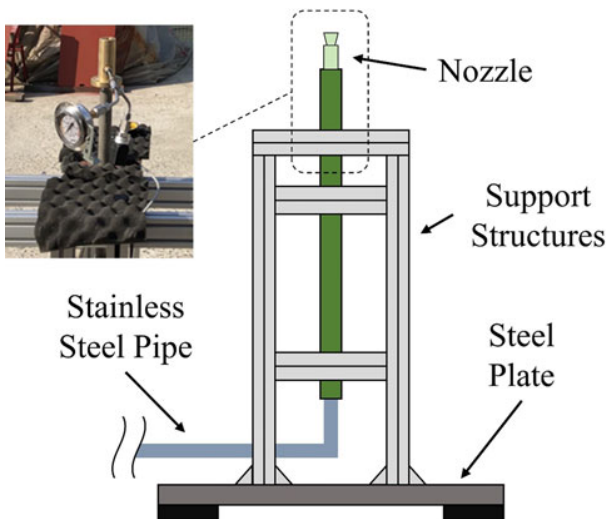

(b)

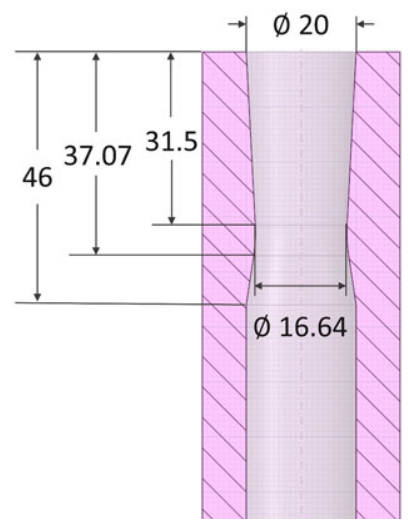

Figure 2. Schematics of the (a) experimental stands and (b) nozzle cross section. All units in millimeters.

the setups of the numerical prediction. Results of both measurement and prediction are presented in Section 4 with a discussion on noise characteristics and discrepancies.

\subsection{Acoustic measurement}

\subsection{Experimental setup}

Acoustic investigations are conducted for a free jet passing through an axisymmetric nozzle. Figure 2 illustrates the outdoor experiment stand for acoustic measurements. As depicted in the figure, a free jet is ejected upward from the ground to minimise the ground effect. The support structures and steel plates with sound absorbing materials are attached to fix the experimental setup. At the end of the pipe, a bundle of gas cylinders are connected for successive experiments and filled with air by a high-pressure compressor. Table 1 summarises the specifications of the nozzle. The diameter of the small-scale nozzle is $D_{e}=20 \mathrm{~mm}$, which is the reference length scale of this study. The nozzle has a converging-diverging shape, in which the area ratio of the nozzle throat to the exit is specified for the corresponding designed exit Mach number of $\mathbf{M}_{d}=1.81$. The fully expanded jet Mach number is $\mathbf{M}_{j}=1.88$ according to its isentropic relation with a given nozzle pressure ratio of 6.5 .

\subsection{Microphone arrays}

Quarter-inch free-field microphones are employed for the acoustic measurement (GRAS 40BE and 46BF, GRAS Sound and Vibration, Copenhagen, Denmark). A digital acquisition system is employed for the experiment (NI-9223, National Instruments, Austin, Texas), and the sampling frequency is set to $200 \mathrm{kHz}$. The microphone arrays are shown in Fig. 3. Linear microphone arrays are arranged parallel to the jet direction. Twelve microphones are equally spaced at intervals of $2.5 D_{e}$. The vertical distances 
Table 2. Observation angle $\theta$ in terms of the linear microphone array position $x$

\begin{tabular}{lccccccccccccc}
\hline Position $\left[\boldsymbol{D}_{e}\right.$ ]. & $x$ & 0.0 & 2.5 & 5 & 7.5 & 10.0 & 12.5 & 15.0 & 17.5 & 20.0 & 22.5 & 25.0 & 27.5 \\
Angle [ $\left.{ }^{\circ}\right]$. & $\theta$ & 142.4 & 136.4 & 128.7 & 118.8 & 106.7 & 92.9 & 78.8 & 65.8 & 55.0 & 46.5 & 39.8 & 34.6 \\
\hline
\end{tabular}

(a)

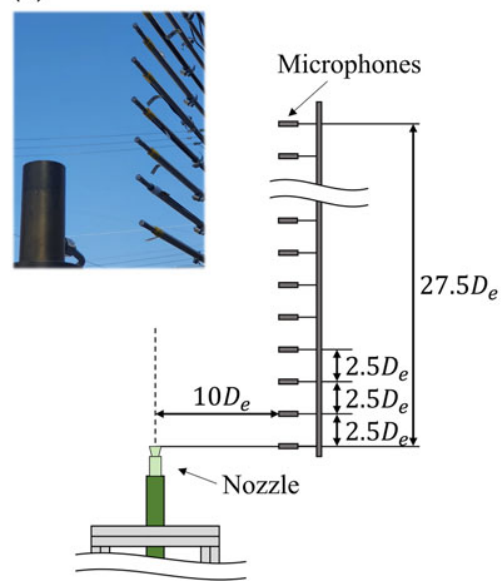

(b)

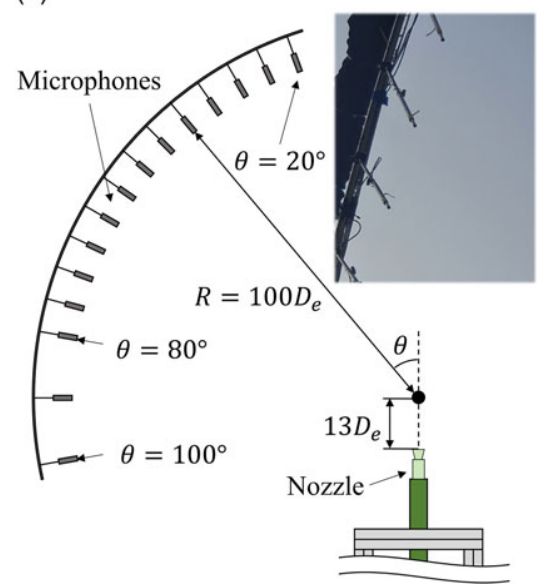

Figure 3. (a) Linear and (b) circular microphone arrays.

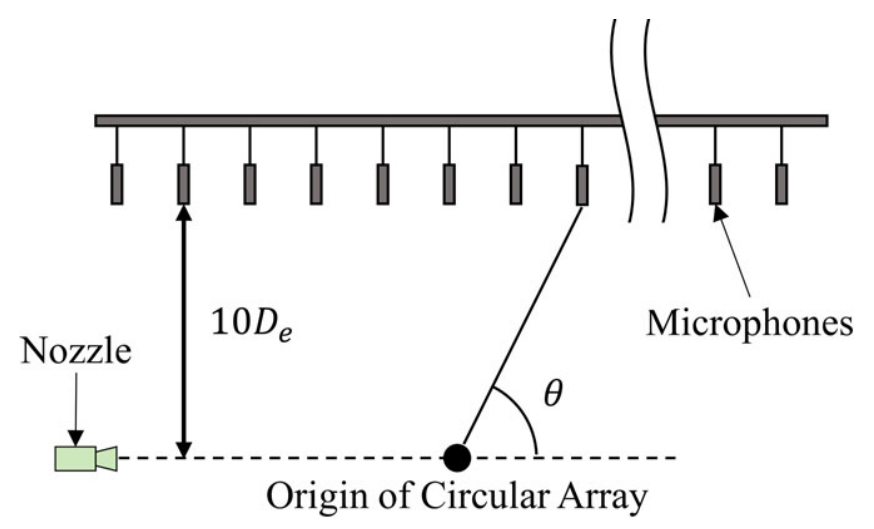

Figure 4. Descriptions of the linear microphone arrays in terms of an observation angle $\theta$.

from the nozzle exit plane to the microphones are distributed from $0 D_{e}$ to $27.5 D_{e}$. The horizontal distance between the nozzle centerline and the linear arrays is $10 D_{e}$. The directivity patterns of the noise are measured using circular arrays of 15 microphones at a radius of $100 D_{e}$. The centre of the array arc is selected to be $13 D_{e}$ away from the nozzle exit plane so that it is located at the point of maximum radiation. This refers to the results of Gee et al. [30], who measured the source region of a Mach 1.81 unheated jet using a phase and amplitude gradient estimator. For $\theta$ between $20^{\circ}$ and $80^{\circ}$, which denotes the angle between the microphones and jet plume axis, the arc of the microphones is separated by a $5^{\circ}$ increment. The increment of the remaining microphone arc is $10^{\circ}$. The purpose of these microphone concentrations at an acute angle is to capture the directivities of the jet noise by Mach wave radiation.

For the convenience of later discussion, Fig. 4 and Table 2 illustrate the angle between the jet plume and the observation location with respect to the origin of the circular array. 


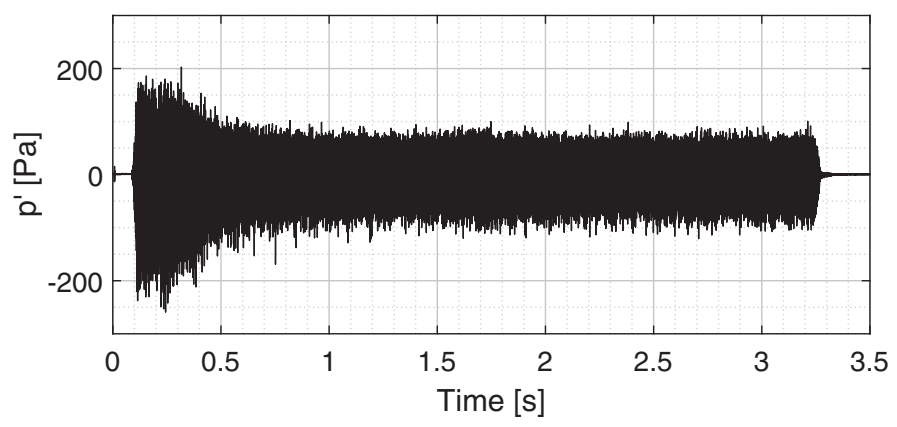

Figure 5. Acoustic pressure with regard to time at the circular microphone at $\theta=75^{\circ}$.

(a)

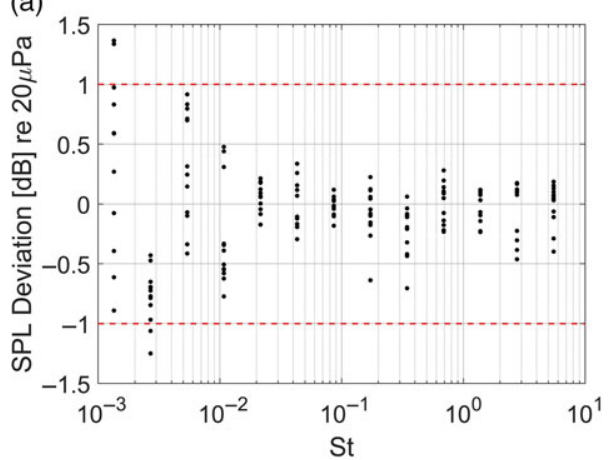

(b)

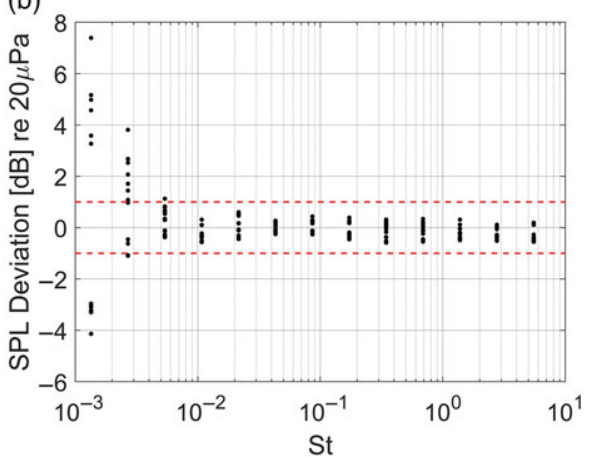

Figure 6. Maximum deviation of 1/1 octave-band SPL for (a) the linear and (b) the circular microphone arrays. Red dashed line indicates $\pm 1 d B$.

\subsection{Evaluation of the measurement}

Figure 5 illustrates the time-varying pressure fluctuations in the jet noise acquired by the microphone arrays. The envelope of the acoustic pressure is observed to stabilise within a short period of time after an initial jet ejection, and the duration corresponding to such an envelope is used for acoustical investigation using a fast Fourier transform.

The reproducibility of the experimental setup is investigated in detail using frequency domain spectra. Figure 6 shows the maximum deviation of the 1/1 octave-band SPL for all the microphones. As shown, the SPL deviations remain within $\pm 1 \mathrm{~dB}$ for $S t=f D_{e} U_{e}>0.0038$ and $S t>0.0076$ for measurements by the linear and circular microphone arrays, respectively. The acoustic measurements were conducted via outdoor tests; however, the results showed sufficient reproducibility, and they validated the current experimental setup.

The background noise is another important factor to be evaluated for the outdoor test [14]. The background noise is affected by the various factors such as the wind speed and humidity. In the present measurement, the wind speed varies from 1.7 to $3.2 \mathrm{~m} / \mathrm{s}$ and the humidity does from $32 \%$ to $36 \%$. First of all, the effect of the background noise may be mitigated by excluding the lower frequency range which dominates the background noise [31] and has large uncertainty as shown in Fig. 6. Therefore, the lower frequency band of $S t \leq 0.0076$ is to be excluded in the present analysis. For the remaining frequency range, the band-limited ( $S t \geq 0.0076$ ) overall sound pressure level (OASPL) of the background noise is measured for all microphones before and after the jet noise measurement. While OASPL of the jet noise is between 121.7 and $146.5 \mathrm{~dB}$ for the same frequency band, that for the background noise is between 
(a)

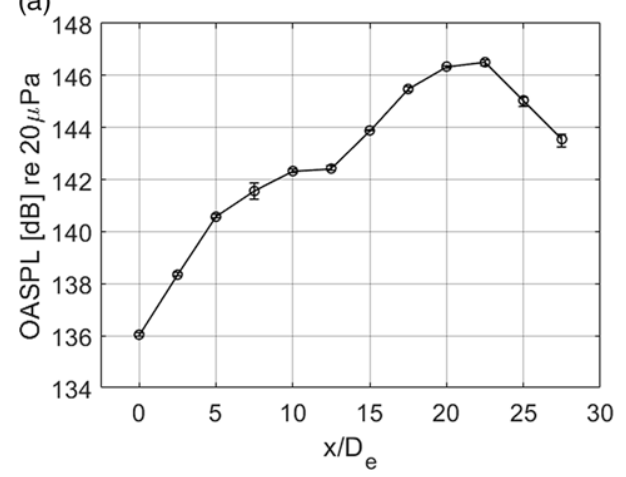

(b)

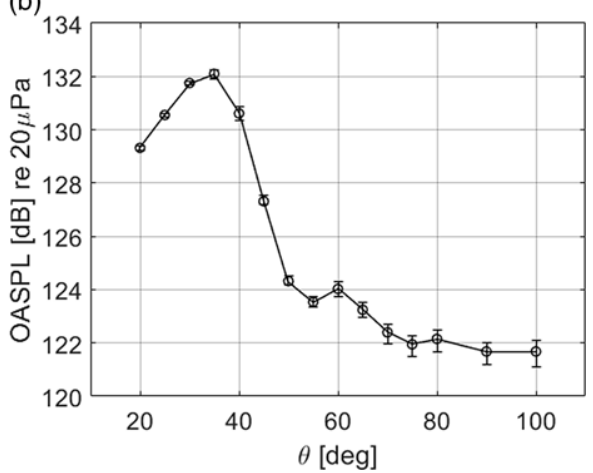

Figure 7. Band-limited (St $>0.0076$ ) OASPL distribution for the (a) linear and (b) circular microphone arrays.

75.2 and $77.8 \mathrm{~dB}$. Therefore, when considering the signal-to-noise ratio, the background noise does not significantly affect the reliability of the jet noise measurement.

Figure 7 shows the OASPL of the jet noise obtained by the microphone arrays. The trends of OASPL can be explained by the turbulent mixing noise, which primarily dominates the supersonic jets and consists of two sources described by Tam et al. [32]: large- and fine-scale turbulent structures. The large-scale turbulent structures are stochastically equivalent to the instability waves, and their relatively supersonic propagation through downstream yields Mach waves. Such Mach waves are usually expressed by wavy wall model which estimates the properties of the directional intense Mach radiation. In contrast, the fine-scale turbulent structures are of low intensity and omni-directionality. They predominate the upstream region where the directional Mach radiation does not prevail.

The OASPL of the linear microphone array generally increases from the measurement location of $0 D_{e}$, as the observation location approaches the main lobe of the large-scale turbulent structures. Then, despite approaching the main lobe, the OASPL starts to decrease beyond the peak OASPL of 146.5dB at the location of $x=22.5 D_{e}$, because of the significantly increased distance between the sound source and observation location. The similar OASPL trend can be found from the study of Vaughn et al. [33], in which the linear microphone array was employed to measure the noise of an ideally expanded jet of $\mathrm{M}_{e}=1.8$.

The effect of the directional Mach radiation is found more clearly by the circular microphone array in which the microphones are at equal distance from the origin. The distinct directivity of the noise is captured at an angle $\theta$ of $35^{\circ}$, where the peak OASPL is $132.1 \mathrm{~dB}$. As the observation location moves far from the angle of peak radiation, OASPL decreases and becomes flat after $\theta \leq 75^{\circ}$, where the finescale turbulence structures become dominating. The peak radiation angle can be compared against that by Gee et al. [30] (between $30^{\circ}$ and $35^{\circ}$ ), who analysed acoustic characteristics of an unheated smallscale jet of $\mathbf{M}_{e}=1.81$ and Oertel's convective Mach number [34] of $\mathbf{M}_{c o}=\left(U_{j}+0.5 c_{j}\right) /\left(c_{j}+c_{a}\right)=1.01$. Considering that the convective Mach number in this paper is $\mathbf{M}_{c o}=1.04$ and that the interval of the microphone array is $5^{\circ}$ for both measurements, it can be assumed that the current results yield a reasonable radiation angle.

The sensitivity of SPL of each 1/1 octave-band is analysed in terms of the linear and circular positions. The sensitivity is represented by the difference of SPL between the adjacent positions, which is divided by an increment of axial distance $x$ and observation angle $\theta$ for the linear and circular microphone arrays, respectively.

Figure 8 shows the results of sensitivity analysis in terms of the linear microphone arrays. Since the distance between the linear microphone and the point of maximum radiation, which is assumed to be $x=13 D_{e}$ [30], varies in terms of the linear location $x$, the SPL variation shows rather complicated trends. For the lower frequency band $(S t<0.24)$, the SPL increase in terms of the axial position for almost of 


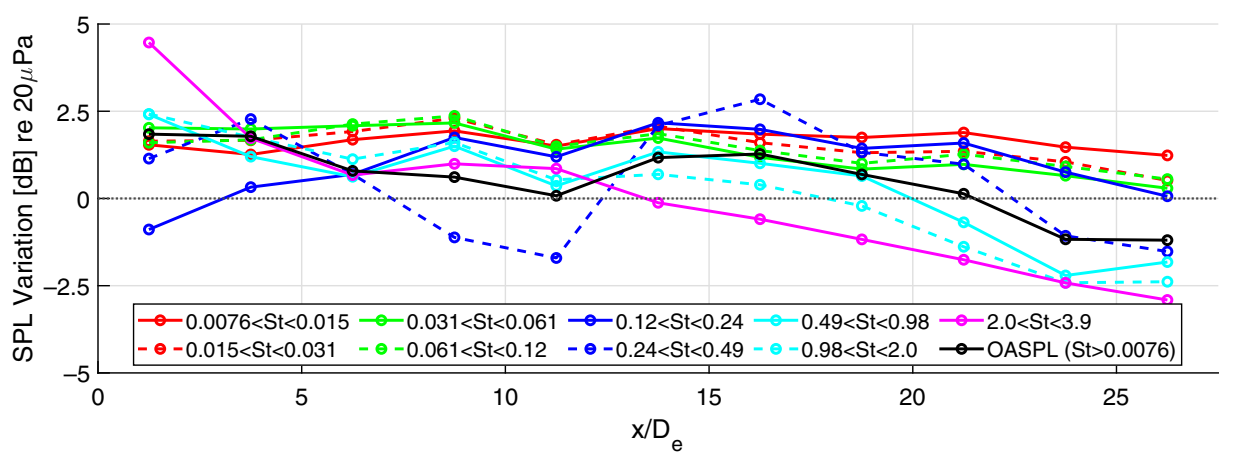

Figure 8. Variation of the $1 / 1$ octave-band SPL per unit characteristic distance $\left(x=1 D_{e}\right)$ for the linear microphone arrays.

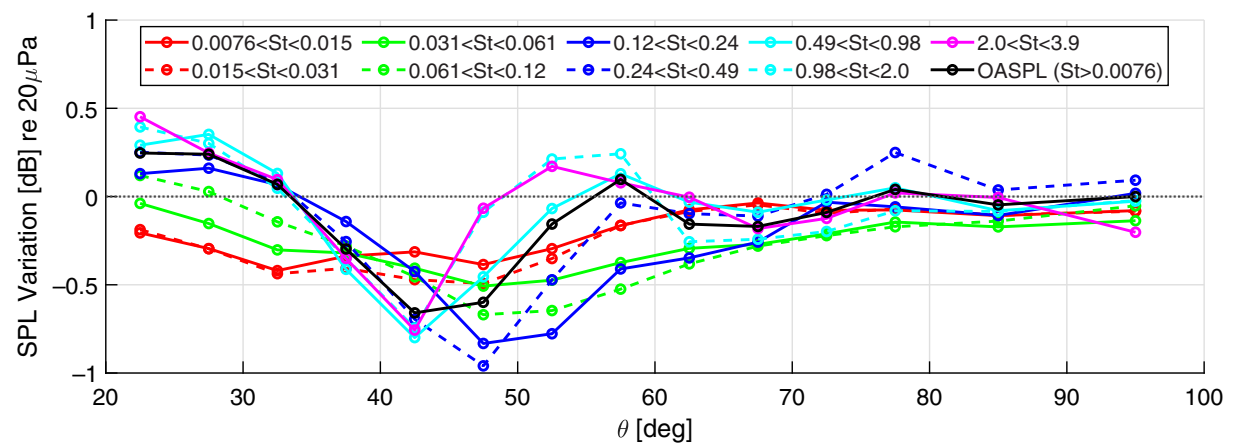

Figure 9. Variation of the $1 / 1$ octave-band SPL per unit observation angle $\left(\theta=1^{\circ}\right)$ for the circular microphone arrays.

microphones in spite of an increase of the distance from the sound source in the downstream $\left(x>13 D_{e}\right)$. Such trend is cause by the directional large-scale turbulence structures of relatively low peak radiation frequency. For higher frequency regime, the SPL initially increases, and decreases as the position moves toward downstream. The notable negative SPL variation is found in $S t>2.0$, which is possibly due to the atmospheric absorption for the high frequency noise. For the circular microphone array of equal distance (Fig. 9), the SPL variation approaches zero value for the large observation angle $\left(\theta>60^{\circ}\right)$, as the omni-directional fine-scale turbulence structures become dominating. SPL variation at the octaveband of $S t>0.031$ varies from the positive to negative value near an angle of $\theta>35^{\circ}$, which is the peak radiation angle determined by the OASPL distribution. The SPL variation of remaining frequency band $(S t<0.031)$ shows the steady negative value, which is possibly contributed by the fine-scale turbulence structures before the sharp frequency region of large-scale ones.

\subsection{The similarity spectra}

The broadband spectra of the measured noise are to be compared against the similarity spectra developed by Tam et al. [32]. They investigated the self similarity of the jet noise by examining the measured results of NASA Langley Research Center Jet Noise Laboratory. The spectra are divided into the largescale strucutre (LSS) and fine-scale strucutre (LSS) spectra, which represent the main behaviour of the turbulent mixing noise. Including the study by Tam et al. [32], successful comparison between the 
(a)

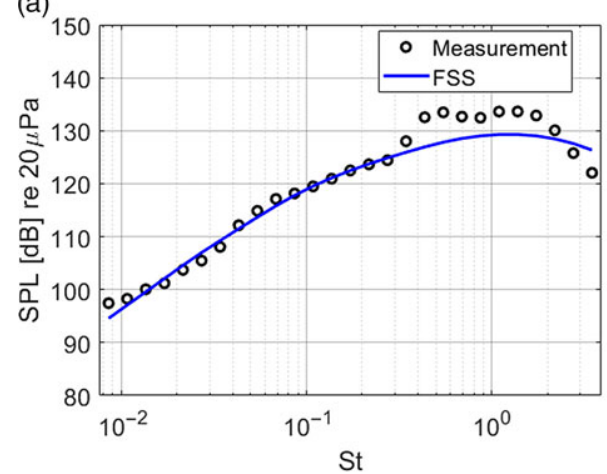

(c)

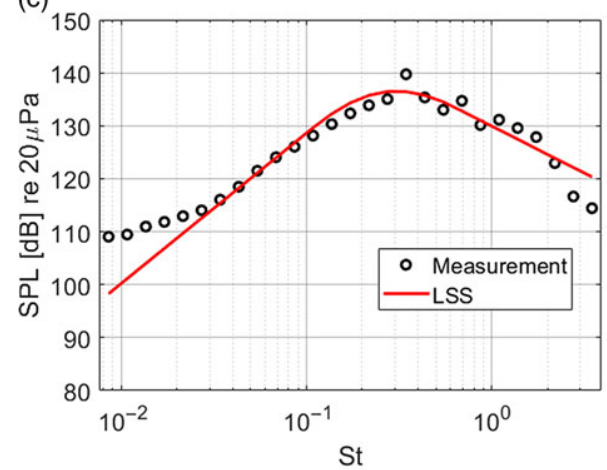

(e)

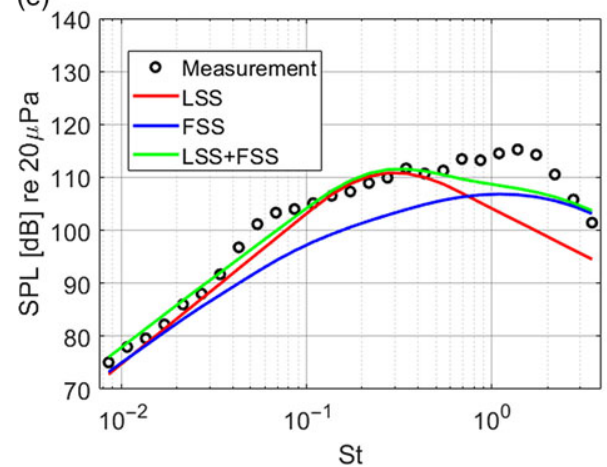

(b)

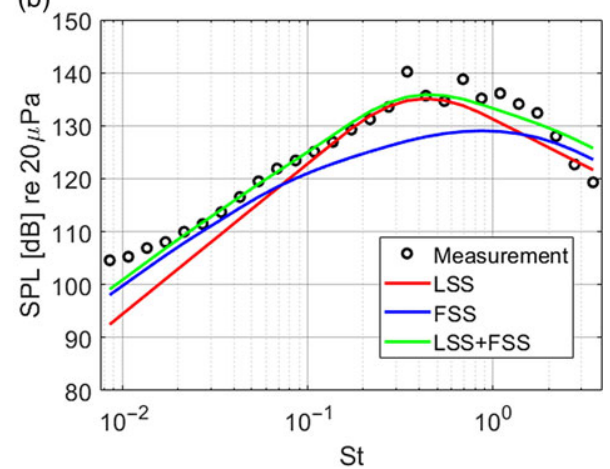

(d)

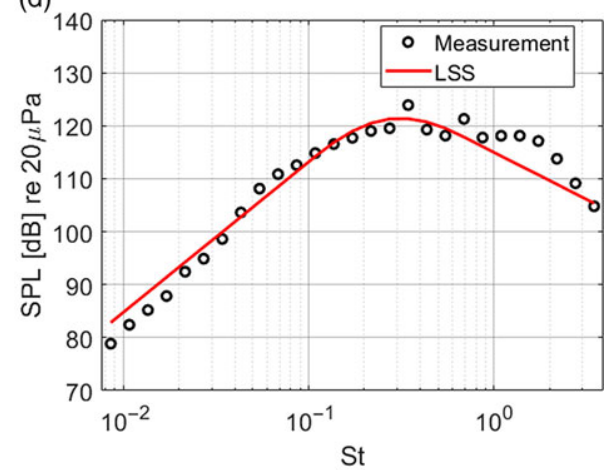

(f)

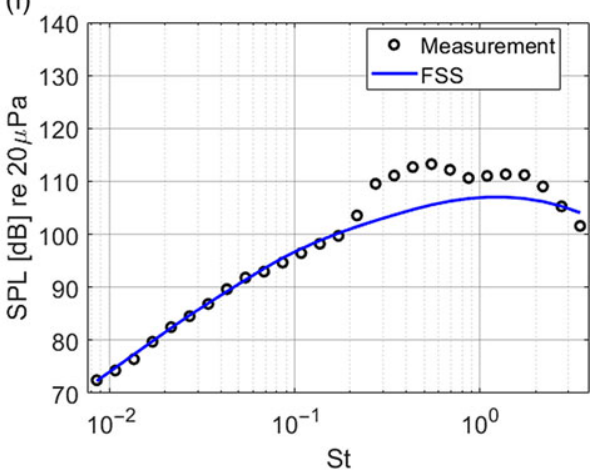

Figure 10. One-third octave-band SPL at the linear array locations of (a) $x=12.5 D_{e}\left(\theta=92.9^{\circ}\right)$, (b) $x=20.0 D_{e}\left(\theta=55.0^{\circ}\right),(c) x=25.0 D_{e}\left(\theta=39.8^{\circ}\right)$, and circular array locations of $(d) \theta=40^{\circ},(e)$ $\theta=55^{\circ},(f) \theta=90^{\circ}$.

similarity spectra and measured results was obtained for the wide variety of jet noise: unheated to heated, near- to far-field, small- to full-scale and subsonic to supersonic [33, 35, 36].

Following the previous studies [35,36], both the similarity spectra and the measured results are analysed on the $1 / 3$ octave-band. For $\theta \leq 45^{\circ}$, LSS spectrum of the narrow and intense nature is employed. In contrast, the broad FSS spectrum is used for $\theta \geq 60^{\circ}$. The LSS and FSS spectra are superposed in the remaining observation angles $\left(45^{\circ}<\theta<60^{\circ}\right)$. The empirical formulation of LSS and FSS spectra was found in Tam et al. [37]

As shown in Fig. 10, the similarity spectra are properly fitted to the experimental results. In addition, the peak frequency of LSS spectrum is fitted to be $f=0.192 c_{a} / D_{j}$ at $\theta=20^{\circ}$, which follows the asymptotic frequency of $f=0.19 c_{a} / D_{j}$ observed by Tam et al. [32], for the given velocity ratio of $U_{j} / c_{a}=1.48$. 


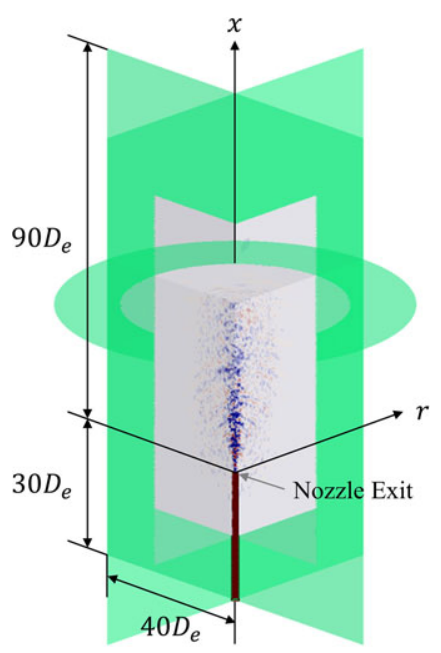

Figure 11. Visualisation of the present CFD domain. Outer transparent green zone is the buffer zone.

However, the discrepancies are also observed for several frequency regimes. In the linear arrays, the microphone measures the higher SPL than the similarity spectra in the lower frequency band. It is possibly due to the hydrodynamic field [33], which decays evanescently in the far-field. Also, the presence of the broadband shock-associated noise of an imperfectly expanded jet generates the discrepancies of large humps in the higher frequency region. The outdoor condition and the atmospheric attenuation may be the other causes for the discrepancies. Nevertheless, the fitted similarity spectra provide the proper representation of the present measurements.

\subsection{Numerical prediction}

\subsection{Numerical setup}

Three-dimensional compressible Navier-Stokes equations are solved using a DES, including the flow field of the nozzle interior. ANSYS Fluent [38], which is based on the finite volume method, is employed for the computation. A Spalart-Allmaras (SA)-based DES is used with a calibration coefficient of $C_{D E S}=0.65$, which determines the DES length scale. Among the pressure-based approaches, the pressure-velocity-coupled algorithm is utilised. There is the pressure-velocity-segregated one which is a memory-efficient alternative to solve the governing equations in a separated manner. However, the coupled algorithm, which solves the continuity and momentum equations in a coupled manner, is selected for the convergence speed and numerical stability despite of the increased memory requirement. For the time integration, the implicit bounded second-order differencing scheme is chosen. The third-order monotonic upstream centered schemes for conservation laws are applied for the spatial discretisation of the momentum and energy equations, modified turbulent viscosity, and density. A conventional secondorder scheme is used for the spatial discretisation of the pressure, and the Green Gauss node-based method is used for gradient computations.

Figure 11 depicts the three-dimensional computational domains of the current CFD simulation. The axisymmetric domain extends by $90 D_{e}$ downstream, $30 D_{e}$ upstream, and $40 D_{e}$ laterally. Non-reflective conditions are imposed for the overall outer boundaries with an atmospheric pressure of 101,325Pa. The adjacent region of the outer boundary consists of a buffer zone with an intentionally stretched grid. Henceforth, the specific location of the computational domain is indicated by the cylindrical coordinate system in Fig. 11.

The flow field is discretised into hexahedral meshes of approximately 55 million grid points, which are created by the blocking procedures in ANSYS ICEM [38]. The grid refinement is focused on the 
(a)

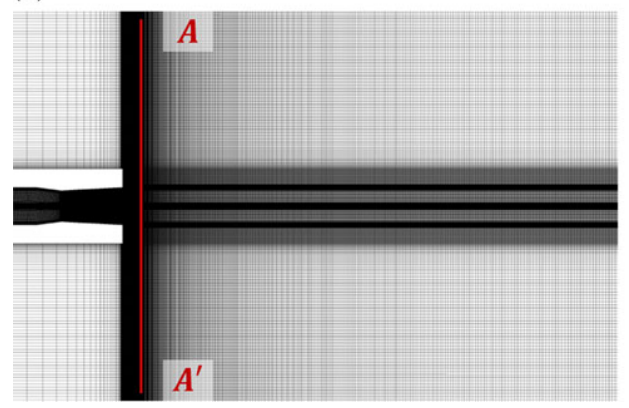

(b)

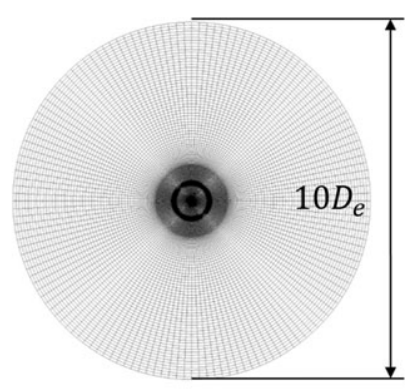

Figure 12. Grid distributions near the nozzle. (a) Cross section through the jet centreline $\left(\overline{A A^{\prime}}=10 D_{e}\right)$. (b) Circular cross section of $\overline{A A^{\prime}}\left(x=0.5 D_{e}\right)$.

inner wall of the nozzle, near the nozzle outlet, and on the jet plume. Figure 12 shows the overall grid distribution near the nozzle exit. At the nozzle wall, the first grid spacing is $0.00015 D_{e}$, which satisfies the maximum dimensionless wall distance of 10. Along the radial direction near the nozzle exit, the minimum grid spacing at the jet shear layer is the same as the first grid spacing of the nozzle wall, and the grid spacing extends beyond. For the nozzle centreline direction, the grid spacing starts with $0.00125 D_{e}$ at the nozzle exit and extends downstream at a ratio of 1.008. However, for the ambient field near the jet plume, computational grids are created by considering the point per wavelength (PPW) of permeable integral surfaces for the acoustic analogy of the Ffowcs-Williams and Hawkings (FW-H) analogy [39]. The setup of the current FW-H is explained in more detail in Section 3.3. Given that the lowest order of the spatial accuracy is 2, the PPW is determined to be 21 [40]. For a cut-off Strouhal number of $S t_{c}=0.4$, which covers the broadband peak of the jet noise while requiring affordable computational cost, the corresponding maximum grid spacing $\Delta_{c}$ is determined to be $0.085 D_{e}$, as follows:

$$
\Delta_{c}=\frac{\lambda_{c}}{P P W}=\frac{1}{P P W} \frac{c_{a}}{f_{c}}=\frac{1}{P P W} \frac{D_{e} c_{a}}{S t_{c} U_{e}},
$$

where $\lambda_{c}$ and $f_{c}$ are the cut-off wavelength and frequency, respectively.

\subsection{Flow computation and post-processing strategy}

For the initial development of the jet plume, the unsteady RANS (URANS) equation is used for a preliminary computation. The SA one-equation turbulence model is selected among the available formulations for the RANS equation. Then, the results are interpolated to DES grid locations, and a few duration executions are processed to eliminate the numerical artifacts induced by differences in the computation models and grid distributions. Finally, computations of the fully established state are performed for the acoustic propagation characteristics. Calculating the total physical time of $720 D_{e} / c_{a}$, which has an exploitable time duration is $550 D_{e} / c_{a}$, takes approximately $2,520 \mathrm{~h}$ by parallel computation using 160 cores of Intel ${ }^{\circledR}$ Xeon ${ }^{\circledR}$ Silver 4114 processors.

An excessive amount of storage is required to maintain all the computational results for the complete time duration; therefore, the results of all the grid locations are stored once every 20 steps. Approximately 60 terabytes of storage space are required to record the relevant results. Then, the flow field quantities at the specific surfaces or locations are extracted via a macro processor in ANSYS and an in-house FORTRAN program.

\subsection{Acoustic analogy}

The FW-H acoustic analogy is utilised with a permeable integral surface to estimate the acoustic propagation for the domain that the CFD computation cannot cover. Based on Formulation 1A of Farassat's 


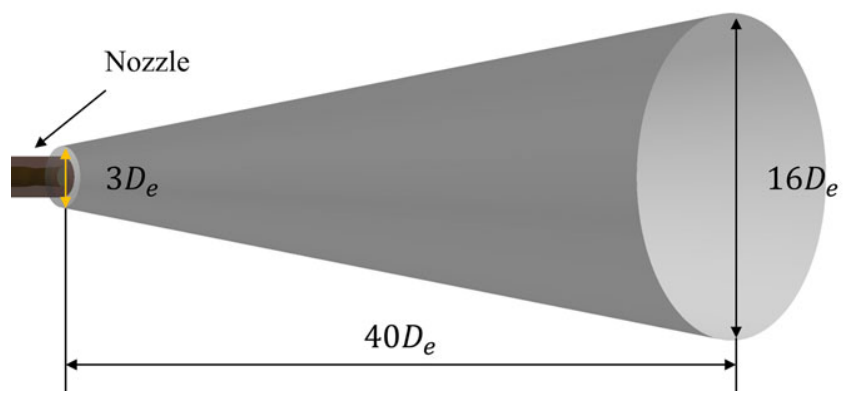

Figure 13. Location of the permeable FW-H surface.

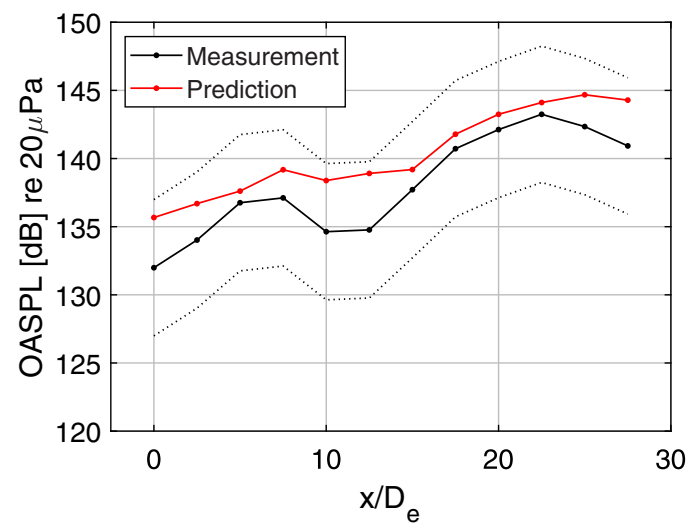

Figure 14. Band-limited $(0.011<S t<0.4)$ OASPL at the linear microphone array. Dotted line indicates $\pm 5 \mathrm{~dB}$ offset of the measured OASPL.

study [41], the acoustic pressure $p^{\prime}(Q, t)$ at stationary location $Q$ and time $t$ from the stationary integral surface is derived as follows:

$$
p^{\prime}(Q, t)=\frac{1}{4 \pi} \iint\left[\frac{(\dot{\rho} u)_{n}}{s}\right]_{r e t} d S+\frac{1}{4 \pi c_{a}} \iint\left[\frac{\dot{L}_{s}}{s}\right]_{r e t} d S+\frac{1}{4 \pi} \iint\left[\frac{L_{s}}{s^{2}}\right]_{r e t} d S,
$$

where $(\dot{\rho} u)_{n}=(\dot{\rho} u)_{I} \hat{n}_{I}$, and $L_{s}=L_{I} \hat{s}_{I}$. The loading noise vector is $L_{I}=P_{I J} \hat{n}_{J}+\rho u_{I} u_{J} \hat{n}_{J}$, where $P_{I J}=$ $p^{\prime} \delta_{I J}-\mu\left(u_{I, J}+u_{J, I}-2 u_{K, K} \delta_{I J} / 3\right)$ for a Stokesian fluid. After separating the time-derivative terms, Equation (2) can be written in the frequency domain using Fourier transforms, as shown in Equation (3)-(5) [42]:

$$
\begin{gathered}
\hat{p}^{\prime}(Q, \omega)=\frac{1}{4 \pi} \iint\left(\hat{F}_{1}(\omega)+\hat{F}_{2}(\omega)\right) \exp \left(\frac{-i \omega s}{c_{a}}\right) d S \\
\hat{F}_{1}(\omega)=i \omega \int_{-\infty}^{\infty}\left(\frac{(\rho u)_{n}}{s}+\frac{L_{s}}{c_{a} s}\right) \exp (-i \omega t) d t \\
\hat{F}_{2}(\omega)=\int_{-\infty}^{\infty}\left(\frac{L_{s}}{s^{2}}\right) \exp (-i \omega t) d t
\end{gathered}
$$

$\hat{p}^{\prime}$ is the time-Fourier-transformed acoustic pressure in the frequency domain. $\hat{F}_{1}$ and $\hat{F}_{2}$ are Fourier transform of the time-differentiated and non-time-differentiated source terms. The effect of the retarded time is reflected by multiplying $\exp \left(-i \omega s / c_{a}\right)$. As suggested by Spalart and Shur [43], Equation (3) 
(a)

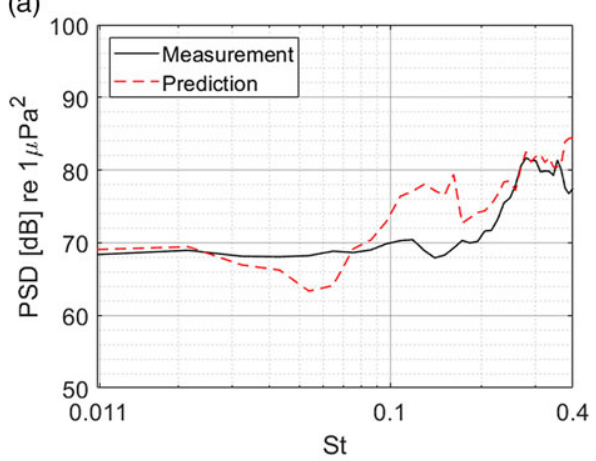

(c)

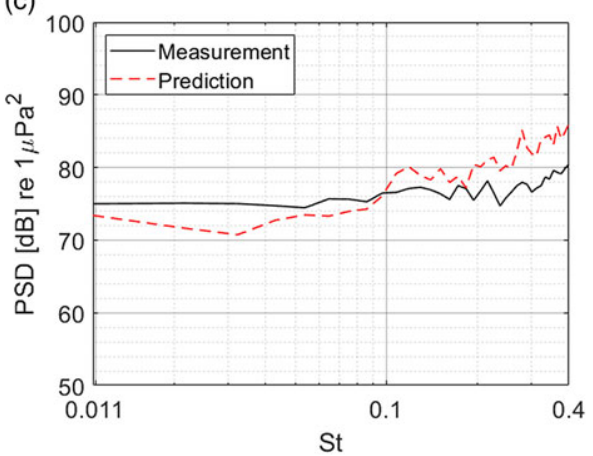

(e)

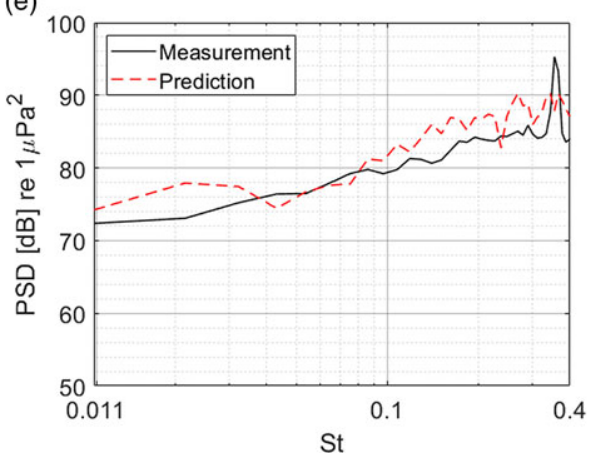

(b)

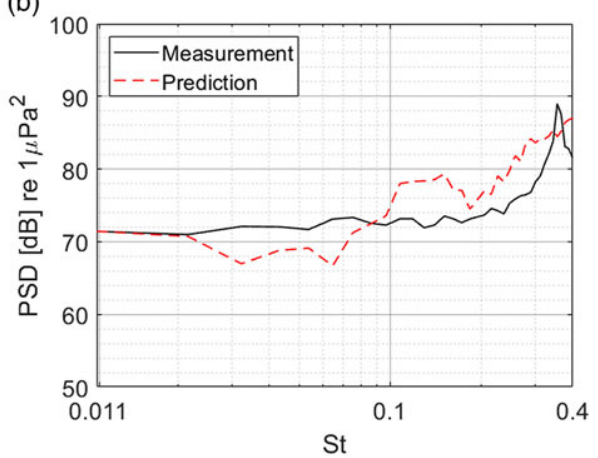

(d)

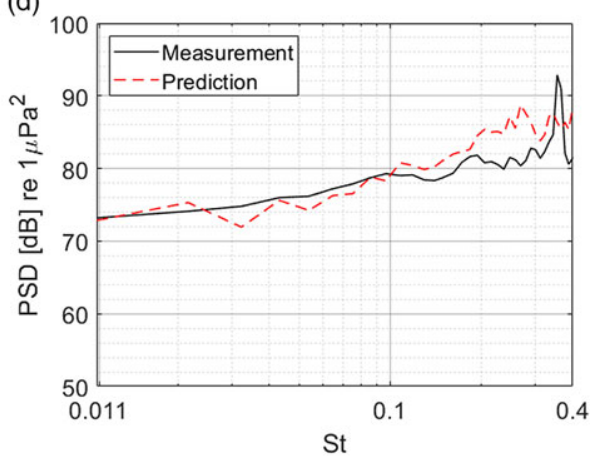

(f)

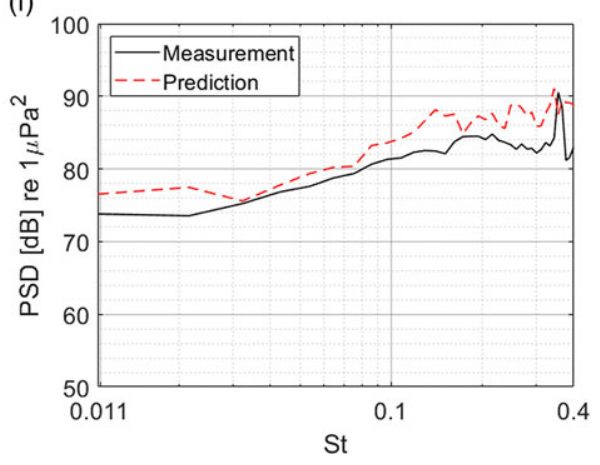

Figure 15. PSD at the linear array locations. (a) $x=2.5 D_{e}\left(\theta=136.4^{\circ}\right)$. (b) $x=7.5 D_{e}\left(\theta=118.8^{\circ}\right)$. (c) $x=12.5 D_{e}\left(\theta=92.9^{\circ}\right)$. (d) $x=17.5 D_{e}\left(\theta=65.8^{\circ}\right)$. (e) $x=22.5 D_{e}\left(\theta=46.5^{\circ}\right)$. (f) $x=27.5 D_{e}$ $\left(\theta=34.6^{\circ}\right)$.

is applied in pressure-based formulation by replacing $\rho$ by $\rho_{a}+p^{\prime} / c_{a}^{2}$. To prevent spectral leakage, a Hanning window is used before the Fourier transforms. The locations of the permeable FW-H surfaces are shown in Fig. 13. The cone-shaped surface extends from the nozzle exit to $40 D_{e}$ downstream. The diameter of the surface is determined to be $3 D_{e}$ and $16 D_{e}$ at the nozzle exit and downstream end, respectively. In addition, because the surface extends sufficiently far downstream, no end cap is employed.

\subsection{Comparision between the acoustic measurement and numerical prediction}

\subsection{Linear microphone array}

The acoustic field at the linear microphone array in Fig. 3(a) is depicted by the band-limited $(0.011<$ $S t<0.4)$ OASPL, as shown in Fig. 14. The lower limit $(S t=0.011)$ is the Strouhal resolution of the subdivided Hanning window, and the higher limit $(S t=0.4)$ corresponds to the cut-off Strouhal number. 


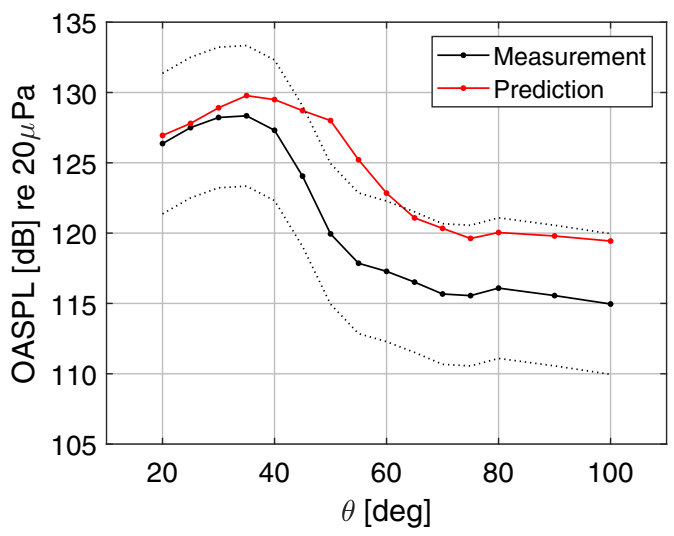

Figure 16. Band-limited $(0.011<S t<0.4)$ OASPL at the circular microphone array. Dotted line indicates $\pm 5 \mathrm{~dB}$ offset of the measured OASPL.

The OASPL of the numerical predictions moderately follows the tendency of the measurements, whereas the maximum overestimation of $4.1 \mathrm{~dB}$ appears at the location of $x=12.5 D_{e}$.

Figure 15 shows the power spectral density (PSD) of the acoustic pressure in some locations. For the measured PSD, a distinct peak of $S t=0.36$ appears at $15 D_{e} \leq x \leq 27.5 D_{e}$, and its magnitude decreases sharply at the locations of $10 D_{e} \leq x \leq 12.5 D_{e}$. Along the upstream, near-plateau profiles are obtained before the respective peak frequencies, and a distinct frequency of $S t=0.36$ slightly reappears at the locations of $0 D_{e} \leq x \leq 7.5 D_{e}$. However, $S t=0.36$ is not currently found to be the screech frequency, because the universal formulation for the screech frequency by Tam et al. [44] rather provides a deviated fundamental screech frequency of $S t=0.19$ for the present jet flow. The other reason is that the relative amplitude of $S t=0.36$ decreases or disappears as the observer moves upstream, where the screech tone usually dominates when it exists. Additionally, another peak frequency of $S t=0.28$ occurs as bumps at the locations of $0 D_{e} \leq x \leq 2.5 D_{e}$. Those bumps of the upstream observers exhibit typical characteristics of broadband shock-associated noise, and they will also be present in the PSD of circular arrays. Overall PSD magnitudes of the lower frequency band increase according to the downstream direction. However, the PSD obtained by numerical prediction is in good agreement for the frequency regime of $S t<0.1$, except for the underestimation at $2.5 D_{e} \leq x \leq 7.5 D_{e}$. For the higher frequency band $(S t>0.1)$, an overprediction of the PSD level is observed.

\subsection{Circular microphone array}

Figure 16 shows the band-limited OASPL of the circular microphone array in Fig. 3(b). The maximum OASPL occurs at $\theta=35^{\circ}$, the direction of which is expected to be that of the Mach radiation for both measurements and numerical predictions. The peak frequencies are $S t=0.36$ and $S t=0.37$ for measurements and numerical prediction, respectively. After $\theta=35^{\circ}$, discrepancies between the measurements and numerical predictions become larger, and a maximum deviation of $8.1 \mathrm{~dB}$ occurs at $\theta=50^{\circ}$. However, the OASPL of numerical predictions generally follows the trends of the experiments; both OASPL distributions exhibit the same peak angle, decrease after $\theta=50^{\circ}$, and become flat after $\theta=75^{\circ}$.

In more detail, Fig. 17 shows the PSD of the circular microphone array. For the measurements, a broadband peak frequency of $S t=0.36$ appears near the peak radiation angle, and it becomes blunt as the observation angle $\theta$ increases. Similar to Section 4.1, nearly flat PSD profiles are observed before peak frequencies at higher angles. Additionally, their overall PSD magnitude decreases along the upstream direction, while small bumps of broadband shock-associated noise appear in the high-frequency range. 
(a)

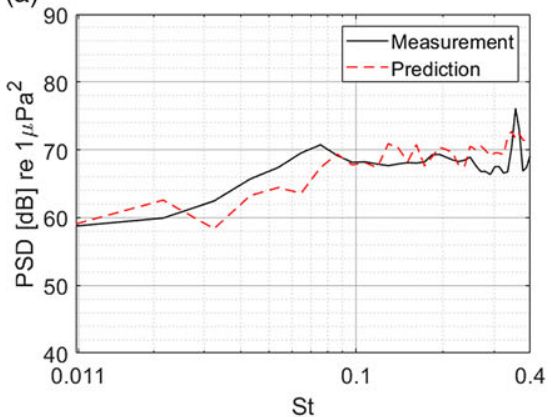

(c)

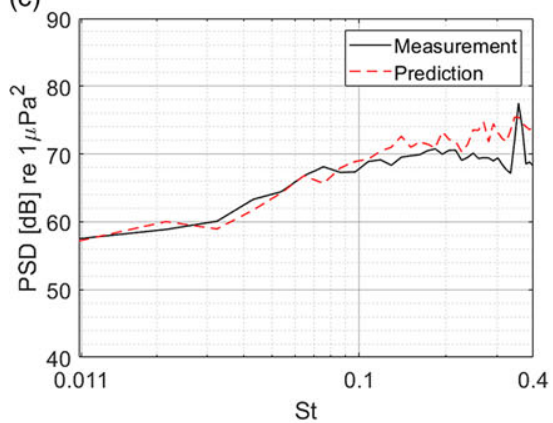

(e)

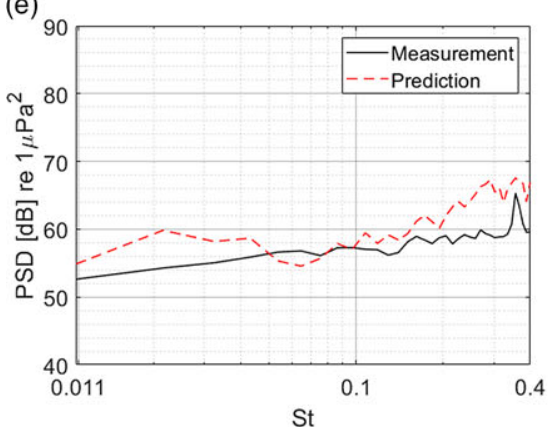

(g)

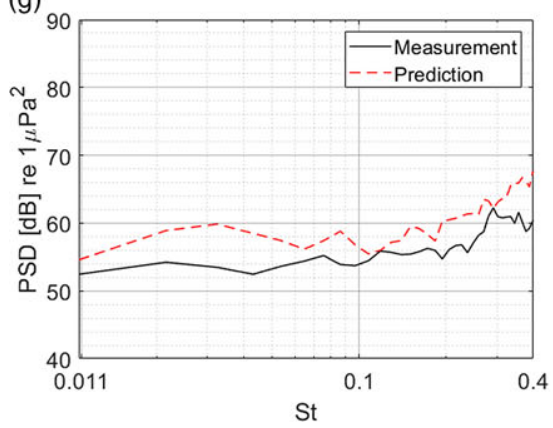

(b)

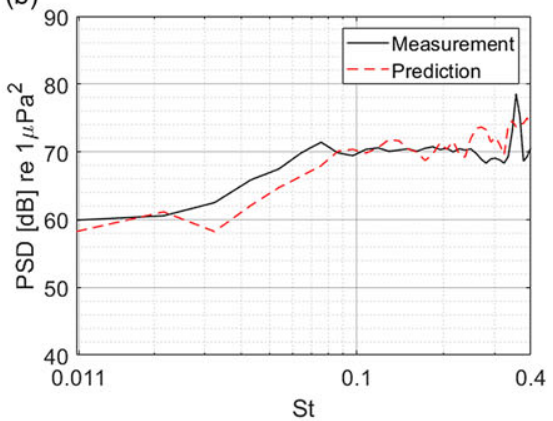

(d)

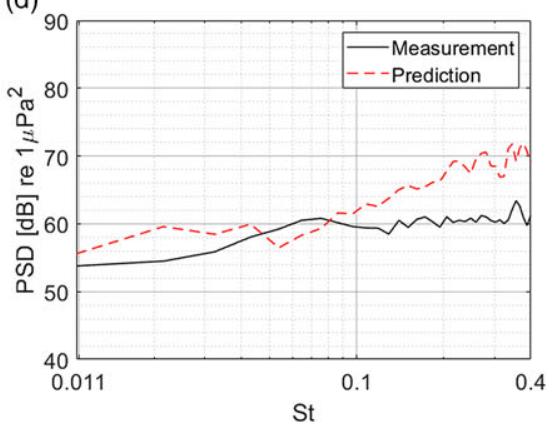

(f)

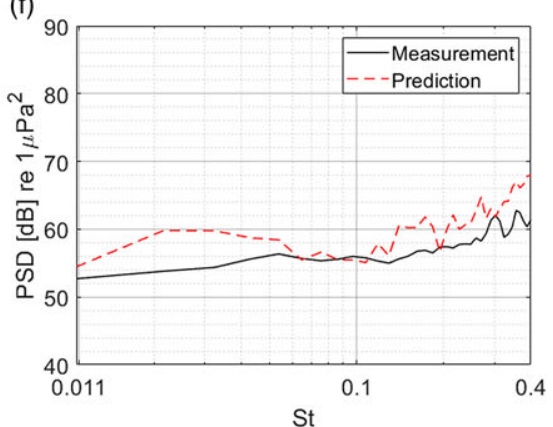

(h)

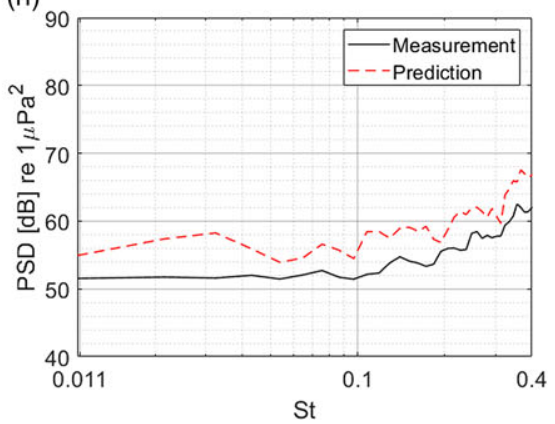

Figure 17. PSD at the circular array locations. (a) $\theta=20^{\circ}$. (b) $\theta=30^{\circ}$. (c) $\theta=40^{\circ}$. (d) $\theta=55^{\circ}$. (e) $\theta=65^{\circ}$. (f) $\theta=80^{\circ}$. (g) $\theta=90^{\circ}$. (h) $\theta=100^{\circ}$.

Numerical predictions are in good agreement with measurements at the location of $\theta \leq 35^{\circ}$. However, overestimations of PSD at $S t>0.1$ occur as the angle increases. For both microphone arrays, current numerical predictions are in good agreement with measurements for downstream observers, for which 
large-scale turbulence structures of Mach wave radiation predominate the background fine-scale turbulence [1]. However, over-estimation of PSD occurs as the observer moves upstream, where fine-scale turbulence becomes dominant. The cause of this is not clear; however, factors such as mesh stretching of the structured grid, spurious noise, and insufficient resolution of the turbulent structures have been suggested as possible causes in previous studies [17, 18].

\subsection{Conclusions}

Acoustic measurements and predictions for an unheated under-expanded free jet were conducted at a fully expanded Mach number of 1.88 with a design Mach number of 1.81. A vertical ejection experiment was conducted with linear and circular microphone arrays, and its reproducibility was validated in terms of the 1/1 octave band SPL. Furthermore, comparison between the similarity spectra of Tam et al. and the measured noise was conducted. For the numerical predictions, DES was performed using a permeable FW-H acoustic analogy.

The experiment indicated noise directivity at an angle of $35^{\circ}$ and characteristics of broadband shockassociated noise. In addition, unexpected noise of discrete intensity was observed at the Strouhal number of 0.36 . The numerical prediction was in good agreement with measurements according to the jet downstream, where a large-scale turbulence structures were expected to dominate. However, overestimations of broadband PSD occurred in the upstream direction, especially at the high-frequency level.

The research experience of this study will be used for future studies on the duct noise of supersonic jets. The noise of a deflector with various duct exit configurations will be investigated using current outdoor experimental stands and numerical predictions.

Acknowledgements. This work was conducted at the High-Speed Vehicle Research Center of the Korea Advanced Institute of Science and Technology (KAIST) with the support of the Defense Acquisition Program Administration (DAPA) and Agency for Defense Development (ADD) under Contract UD170018CD.

\section{References}

[1] Tam, C.K.W. Supersonic jet noise, Annu Rev Fluid Mech, 1995, 27, pp 17-43.

[2] Punekar, J.N., Avital, E. and Li, X. Experimental investigation of nonlinear properties of crackle and screech in supersonic jets, J Acoust Soc Am, June 2017, 141, (6), pp EL567-EL573.

[3] Vaughn, A., Swift, S.H., Gee, K.L., Downing, M., James, M.M. and Wall, A.T. Beamforming of crackle-related events in supersonic jet noise, J Acoust Soc Am, October 2018, 144, (3), p 1671.

[4] Ajaj, R.M., Allegri, G. and Isikveren, A.T. Conceptual design and sizing of airframe panels according to safe-life acoustic fatigue criteria, Aeronaut J, January 2011, 115, (1163), pp 15-27.

[5] Lighthill, M.J. On sound generated aerodynamically I. General theory, Proc R Soc A, March 1952, 211, (1107), pp 564-587.

[6] Lighthill, M.J. On sound generated aerodynamically II. Turbulence as a source of sound, Proc R Soc A, February 1954, 222, (1148), pp 1-32.

[7] Seiner, J.M. Advances in high speed jet aeroacoustics, 9th Aeroacoustics Conference, AIAA-1984-2275, Williamsburg, Virginia, USA, Octobar 1984.

[8] Bailly, C. and Fujii, K. High-speed jet noise, Mech Eng Rev, 2016, 3, (1), pp 1-13.

[9] Eldred, K.M. Acoustic loads generated by the propulsion system, NASA SP-8072, 1971.

[10] Haynes, J., and Kenny R. Modifications to the NASA SP-8072 distributed source method II for Ares I lift-off environment predictions, 15th AIAA/CEAS Aeroacoustics Conference (30th AIAA Aeroacoustics Conference), AIAA-2009-3160, Miami, Florida, USA, May 2009.

[11] Varnier, J. Experimental study and simulation of rocket engine freejet noise, AIAA J, Octobar 2001, 39, (10), pp 1851-1859.

[12] Kandula, M. Near-field acoustics of clustered rocket engine, J Sound Vib, January 2008, 309, (3-5), pp 852-857.

[13] Barbarino, M., Adamo, F.P., Bianco, D. and Bartonccini D. Hybrid BEM/empirical approach for scattering of correlated sources in rocket noise prediction, J Sound Vib, September 2017, 403, pp 90-103.

[14] Fukuda, K., Tsutsumi, S., Fujii, K., Ui, K., Ishii, T., Oinuma, H., Kazawa, J. and Minesugi, K. Acoustic measurement and prediction of solid rockets in static firing tests, 15th AIAA/CEAS Aeroacoustics Conference (30th AIAA Aeroacoustics Conference), AIAA-2009-3368, Miami, Florida, USA, May 2009.

[15] Mallinson, S.G., Reizes, J.A and Hong, G. An experimental and numerical study of synthetic jet flow, Aeronaut J, January 2001, 105, (1043), pp 41-49.

[16] Bodony, D. and Lele, S. Current status of jet noise predictions using large-eddy simulation, AIAA J, February 2008, 46, (2), pp 364-380. 
[17] Housman, J.A., Stich, G.-D., Kiris, C.C. and Bridges, J. Jet noise prediction using hybrid RANS/LES with structured overset grids, 23rd AIAA/CEAS Aeroacoustics Conference, AIAA-2017-3213, Denver, Colorado, USA, June 2017.

[18] Tsutsumi, S., Ishii, T., Ui, K., Tokudome, S. and Wada, K. Study on acoustic prediction and reduction of Epsilon launch vehicle at liftoff, J Spacecr Rockets, March 2015, 52, (2), pp 350-361.

[19] Tsutsumi, S., Ishii, T., Ui, K., Tokudome, S. and Wada, K. Assessing prediction and reduction technique of lift-off acoustics using Epsilon flight data, 53rd AIAA Aerospace Sciences Meeting, AIAA-2015-1007, Kissimmee, Florida, USA, January 2015.

[20] Kiris, C.C., Housman, J.A., Barad, M.F., Brehm, C., Sozer, E. and Moini-Yekta, S. Computational framework for Launch, Ascent, and Vehicle Aerodynamics (LAVA), Aerosp Sci Technol, August 2016, 55, pp 189-219.

[21] Dougherty, N. and Guest, S.A correlation of scale model and flight aeroacoustic data for the space shuttle vehicle, 9th Aeroacoustics Conference, AIAA-1984-2351, Williamsburg, Virginia, USA, Octobar 1984.

[22] Varnier, J., Piet, J.F., Gely, D., Elias, G. and Radulovic, S. Modeling of the acoustic environment on the Ariane 5 fairing using small scale test data, 2nd AIAA/CEAS Aeroacoustics Conference, AIAA-1996-1721, State College, Pennsylvania, USA, May 1996.

[23] Troutt, T.R. and McLaughlin, D.K. Experiments on the flow and acoustic properties of a moderate-Reynolds-number supersonic jet, J Fluid Mech, March 1982, 116, pp 123-156.

[24] Ishii, T., Tsutsumi, S., Ui, K., Tokudome, S., Ishii, Y., Wada, K. and Nakamura, S. Acoustic measurement of 1:42 scale booster and launch pad, Proc Meet Acoust, May 2014, 18, pp 1-18.

[25] Malbéqui, P., Davy, R. and Bresson, C. Experimental characterization of the acoustics of the future Ariane 6 launch pad, 7th European Conference for Aeronautics and Space Sciences, Milan, Italy, July 2017.

[26] Jiang, C., Han, T., Gao, Z. and Lee, C.-H. A review of impinging jets during rocket launching, Prog Aerosp Sci, August 2019, 109, pp 1-22.

[27] Jang, I., Ohm, W., Joo, H., Shin, S.-J. and Park, J. Sensitivity of acoustic predictions on the mesh size of large-eddy simulation of supersonic jet, J Acoust Soc Am, Octobar 2018, 144, (3), p 1642.

[28] Park, T., Jang, I., Ohm, W., Joo, H., Kang, S., Shin, S.-J and Park, J. Comparison of exhaust duct designs in launch pads for reduction of rocket jet noise, J Acoust Soc Am, November 2019, 146, (4), p 3041.

[29] Kang, S., Joo, H., Shin, S.-J., Park, T., Ohm, W. and Park, J. Acoustic experiment and numerical simulation on unheated supersonic jet flow for a small-scale nozzle, AIAA Scitech 2020 Forum, AIAA-2020-0002, Orlando, Florida, USA, January 2020 .

[30] Gee, K.L., Akamine, M., Okamoto, K., Neilsen, T.B., Cook, M.R., Tsutsumi, S., Teramoto, S. and Okunuki, T. Characterization of supersonics laboratory-scale jet noise with vector acoustic intensity, 23rd AIAA/CEAS Aeroacoustics Conference, AIAA-2017-3519, Denver, Colorado, USA, June 2017.

[31] Albert, D.G. and Decato, S.N. Acoustic and seismic ambient noise measurements in urban and rural areas, Appl Acoust, April 2017, 119, pp 135-143.

[32] Tam, C.K.W., Golebiowski, M. and Seiner, J.M. On the two components of turbulent mixing noise from supersonic jets, Aeroacoustics Conference, AIAA-1996-1716, State College, Pennsylvania, USA, May 1996.

[33] Vaughn, A.B., Neilsen, T.B., Gee, K.L., Okamoto, K. and Akamine, M. Near-field spatial variation in similarity spectra decomposition of a Mach 1.8 laboratory-scale jet, Proc Mtgs Acoust, June 2017, 29, pp 1-10.

[34] Oertel, H. Measured velocity fluctuations inside the mixing layer of a supersonic jet. In: Haase W. (eds) Recent Contributions to Fluid Mechanics, Springer, Berlin, Germany, 1982.

[35] Viswanathan, K. Analysis of the two similarity components of turbulent mixing noise, AIAA J, September 2002, 40, pp 1-10.

[36] Neilsen, T.B., Gee, K.L., Wall, A.T. and James, M.M. Similarity spectra analysis of high-performance jet aircraft noise, $J$ Acoust Soc Am, April 2013, 133, (4), pp 2116-2125.

[37] Tam, C.K.W. and Zaman, K.B.M.Q. Subsonic jet noise from nonaxisymmetric and tabbed nozzles, AIAA J, April 2000, 38, (4), pp 1735-1744.

[38] Anonymous ANSYS, Software Package, Ver. 20.1, ANSYS, Inc., Canonsburg, Pennsylvania, USA, 2020.

[39] Williams, J.E.F. and Hawkings, D.L. Sound generation by turbulence and surfaces in arbitrary motion, Philos Trans R Soc A, May 1969, 264, (1151), pp 321-342.

[40] Wilson, R.V., Demuren, A.O. and Carpenter, M. Higher-order compact schemes for numerical simulation of incompressible flows, NASA/CR-1998-206922, February 1998.

[41] Farassat, F. Derivation of Formulations 1 and 1A of Farassat, NASA/TM-2007-214853, May 2007.

[42] Mendez, S., Shoeybi, M., Lele, S.K. and Moin, P. On the use of the Ffowcs Williams-Hawkings equation to predict far-field jet noise from large-eddy simulations, Int J Aeroacoust, January 2013, 12, (1-2), pp 1-20.

[43] Spalart, P.R. and Shur, M. Variants of Ffowcs Williams-Hawkings equation and their coupling with simulations of hot jets, Int J Aeroacoust, July 2009, 8, (5), pp 477-491.

[44] Tam, C.K.W., Seiner, J.M. and Yu, J.C. Proposed relationship between broadband shock associated noise and screech tones, J Sound Vib, October 1986, 110, (2), pp 309-321.

Cite this article: Kang S., Park T., Joo H., Im S., Jang I., Ohm W. and Shin S.-J. (2022). Acoustic measurement of under-expanded jet and its numerical prediction. The Aeronautical Journal, 126, 1510-1526. https://doi.org/10.1017/aer.2022.3 